\title{
A Review on Ethanopharmacological Importance of Clitoria Ternatea
}

\author{
Sameer Quazi ${ }^{1 *}$, Tejaswini Yogekar ${ }^{2}$
}

${ }^{1}$ Department of Biochemistry, Indian Academy Degree College, Hennur Cross, Bangalore, Karnataka, India

${ }^{2}$ Department of Biotechnology, Indian Academy Degree College, Hennur Cross, Bangalore,Karnataka, India. (560043)

DOI: $10.36347 /$ sajb.2020.v08i03.002

| Received: 02.03.2020 | Accepted: 09.03.2020 | Published: 26.03.2020

*Corresponding author: Sameer Quazi

Abstract

Review Article

Medicinal as well as aromatic plants have been utilized over the ages for their poetncy and negligible side effects. As a result of this, the exploration is at its highest possible peak. Seeing this phenomenon, the climbing plant 'Clitoria ternatea, (CT) coming from the Fabaceae family and frequently referred to as 'Butterfly pea' and 'Shankpushpi'. Traditionally named as Aparajitha pushpam, has been used in Traditional Ayurvedic Medicine, due to its varied usages over centuries as a memory booster, nootropic, antistressor, anxiolytic, antidepressant, anticonvulsant, tranquilizing as well as sedative agent. A vast range of secondary metabolites consisting of triterpenoids, flavanol glycosides, anthocyanins and steroids has been extracted from Clitoria ternatea. Its extracts have a variety of medicinal properties such as antimicrobial, antipyretic, anti-inflammatory, analgesic, diuretic, anesthetic, antidiabetic, insecticidal, blood platelet aggregation-inhibiting as well as vascular smooth muscle tissue relaxing properties. This plant has a longstanding history in Traditional Ayurvedic medication for a number of diseases and the scientific and clinical research studies has reconfirmed those with modern relevance. The plant holds several active constituents like alkaloids, glucosides, flavonoids, saponins, tannins, carbs and so on. This review is an initiative effort to explore the phytochemical components as well as pharmacological studies of Clitoria ternatea, which have remained in professional usage in the Ayurvedic system of medicine along with an essential assessment of its future ethnopharmacological potential in the perspective of many recent findings of importance on the well-known plant species.

Keywords: Clitoria ternatea, Shankpushpi, antioxidant, anti-analgesic, nootropic, sedative agent, anti-depressant. Copyright @ 2020: This is an open-access article distributed under the terms of the Creative Commons Attribution license which permits unrestricted use, distribution, and reproduction in any medium for non-commercial use (NonCommercial, or CC-BY-NC) provided the original author and source are credited

\section{INTRODUCTION}

Large and ever-increasing number of patients use medicinal plants and herbs for better health. Therefore, making it necessary to evaluate of their therapeutic potential, biological properties, and safety. This will be very useful in making informed decisions about their use. Traditional medicinal plants have been useful in development of hundreds of remarkable drugs and biologically active compounds. The plant named Aparajitha pushpam, showed a wide range of pharmacological activities such as, antimicrobial, antioxidant, anticancer, hypolipidemic, cardiovascular, central nervous, respiratory, immunological, anti-inflammatory analgesic, antipyretic and etc. Preliminary phytochemical screening revealed that Clitoria ternatea contained tannins, phlebotomine, carbohydrates, saponins, triterpenoids, phenols, flavonoids, flavanol glycosides, proteins, alkaloids, anthraquinone, anthocyanins, cardiac glycosides, volatile oils and steroids. The plans also exhibited many pharmacological effects including antioxidant, hypolipidemic, anticancer, anti- inflammatory, analgesic, antipyretic, antidiabetic, CNS, antimicrobial, gastro-intestinal antiparasitic, insecticidal and many other pharmacological effects. This review is about highlighting the chemical and pharmacological properties of Clitoria ternatea[1,2].

\section{PHARMACOLOGICAL PROFILE}

Medicinal researches have validated that Clitoria ternatea display a wide range of biological effects, a few of which are really intriguing for future growth. 


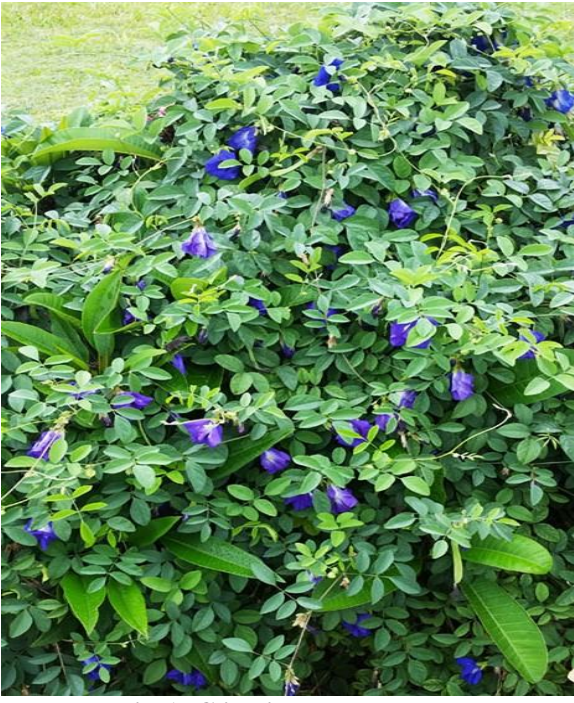

Fig-1: Clitoria ternatea Bush

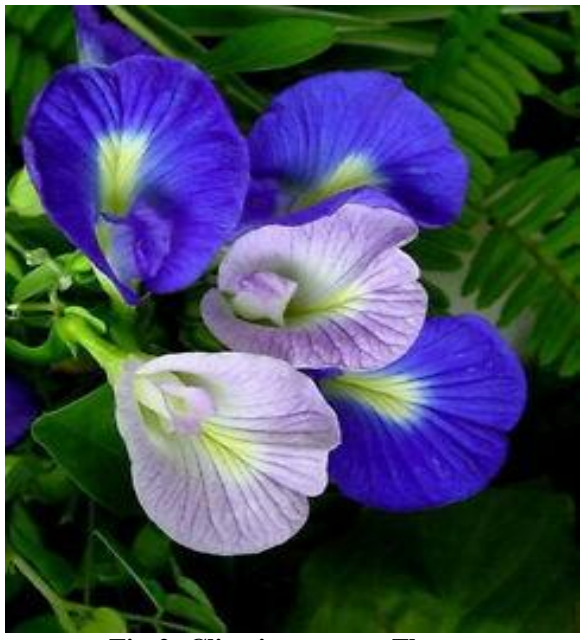

Fig-2: Clitoria ternatea Flower

\section{Memory Boost Activity}

The oral consumption of Clitoria ternatea root extract at doses dramatically enhanced memory in rats. The alcoholic extract of aerial parts and roots of Clitoria ternatea were reported to attenuate aftershock effects of memory loss. The acetylcholine $(\mathrm{AcH})$ content of the entire brain and acetyl cholinesterase activity at various regions of the rat brain, meaning, cerebral cortex, mid-brain, medulla oblongata as well as cerebellum were assessed. It was recommended that a boost in $(\mathrm{AcH})$ in rat hippocampus might be the neurochemical basis for enhanced learning and memory. Rai et al.[3-6] by utilizing passive avoidance test and spatial learning $\mathrm{T}$-maze too have revealed that the aqueous root extract of Clitoria ternatea boosts memory in rats. In an additional reported research, the result of aqueous root extract on the dendritic cytoarchitecture of nerve cells of amygdale was studies. This improved dendritic arborization of amygdaloidal nerve cells correlates with the increased passive avoidance learning and memory in the rats treated with Clitoria ternatea.

\section{Anti-inflammatory and Anti-analgesic Activity}

The research study was aimed at obtaining the anti-inflammatory activity of the methanolic extract from the roots of Clitoria ternatea, making use of rat models. In the same research study, the ethanolic extract too was reviewed for analgesic activity in mice with the acetic acid-induced twisting response and mechanical stimuli by tail clip method.

\section{Anti-Pyretic Activity}

In another research, the methanol extract of Clitoria ternatea was evaluated for its anti-pyretic capacity in albino rats. The anti-pyretic effect of the extract was comparable to that of paracetamol (PCM) (150 mg/kg b.w. p.o) a common antipyretic agent.

\section{Anti-Epileptic Activity}

Methanol extract from the aerial parts of Clitoria ternatea was examined by making use of pentylenetetrazol (PTZ) and Maximum Electro-shock (MES)- induced seizures in mice at the dosage of 100 $\mathrm{mg} / \mathrm{kg}$ p.o. Clitoria ternatea considerably delayed the beginning of convulsions and also shortened the time period of tonic hind limb extension in MES-induced convulsions.

\section{Antioxidative Activity}

It has been clearly stated that oxidative stress is amongst the major causative factors of several degenerative and chronic diseases. Clitoria ternatea petals have been recognized to have anti-oxidant activity. Extract of Clitoria ternatea flowers are used in Thailand as a part of cosmetics and the chemical composition of the flower recommend that they might have antioxidant activity. Aqueous extracts of Clitoria ternatea revealed to have stronger anti-oxidant activity as compared to ethanol extracts. The antioxidant potential of Clitoria ternatea extract in aqueous form was reviewed by determining the level of enzymatic and non-enzymatic anti-oxidants. In-vitro antioxidant capability was likewise established by making use of various assays such as Ferric reducing power assay (FRAP), Reducing Activity assay, diphenypicrylhydrazyl (DPPH) assay and Hydroxyl radical scavenging activity and the results were similar with standard anti-oxidants such as butylated hydroxyl toluene (BHT), ascorbic acid and also rutin.

\section{Anti-Diabetic Activity}

Anti-diabetic researches by oral administration of aqueous extract obtained from Clitoria ternatea leaves (400 mg/kg body weight) and flowers $(400 \mathrm{mg} / \mathrm{kg}$ body weight) for 84 days revealed significant decrease in serum glucose, glycosylated hemoglobin, total cholesterol, triglycerides, urea, creatinine as well as the activity of gluconeogenic enzyme glucose-6-phosphatase, but raised levels of serum insulin, HDL cholesterol, protein, liver and skeletal muscle glycogen content and the activity of glycolytic 
enzyme glucokinase. For all the above biochemical parameters checked out, Clitoria ternatea leaves cured rat revealed to have a little better activity, in comparison to the rats treated by flowers of Clitoria ternatea.

\section{Anti-Blood Platelet Aggregation Activity}

Anthocyanin ternatins D1 obtained from petals of Clitoria ternatea was examined for in-vitro platelet aggregation repression activity in rabbits. The results of various documented research studies revealed to have substantial inhibition of collagen and adenosine diphosphate (ADP) induced aggregation of platelets.

\section{Anesthetic Activity}

The anesthetic effect of alcoholic extract of aerial parts from Clitoria ternatea was studied by Kulkarni et al, making use of corneal anesthetic in rabbits and plexus anesthetic in frogs. The outcomes were nearly as reliable as xylocaine in induced anesthesia.

\section{Anti-anxiety Activity}

The oral administration of Clitoria ternatea $(30 \mathrm{mg} / \mathrm{kg})$ failed to exhibit and significant effect in both the animal models of anxiety. But the oral administration of $(100-400 \mathrm{mg} / \mathrm{kg})$ dose comparatively increased the time spent in the open arm, the time spent in the lit and decreased the duration of time spend in the dark box. The animals treated with $(100 \mathrm{mg} / \mathrm{kg})$ exhibited a remarkable increase in the inflexion ratio and discrimination index that provides evidence for the plant's nootropic activity.

\section{Central Nervous System Depressant Activity}

The extract lowered time needed for occupying the central platform, exhibiting transfer latency, in the elevated plus puzzle (EPM) and raised discrimination index in the object identification/recognition test, showing nootropic activity. It decreased the duration of immobility in tail suspension test, minimized stress and anxiety induced ulcers and reduced the convulsing action of PTZ and MES. Reduced intensity of behavior mediated through serotonin and $\mathrm{AcH}$ was also exhibited by the extract.

\section{Anti-Stress Activity}

The anti-stress activity of airborne parts of Clitoria ternatea was evaluated using cold restrain test (CRS) caused ulcers, lithium-induced head twitches, clonidine-induced hypothermia, salt nitrite-induced breathing apprehension and haloperidol-induced catalepsy in rat and mice.

\section{Larvicidal Activities}

The methanol extract of Clitoria ternatea seed worked efficiently against the larvae of all the 3 species, A. stephensi, A. aegypti and C. quinquefasciatus with LC50 having values 65.2, 154.5 and $54.4 \mathrm{ppm}$. Clitoria ternatea showed one of the most appealing larvicidal activities for mosquitoes.

\section{Proteolytic Activity}

The activity of endopeptidases, hemoglobin ( $\mathrm{pH}$ 3.5) and azocasein ( $\mathrm{pH}$ 6.0), carboxypeptidase benzyloxy carbonyl [CBZ-Phe-Ala (Ph 5.2)], arylamidases lysophosphatidic acid and a-N-benzoyl-L-arginine P-nitro-analide (LPA 7.0) and (BAPA 7.6) were assayed in the seed extract of cotyledons and axis of resting and sprouting seeds of Clitoria ternatea. However, the endopeptidases at $\mathrm{pH} 3.5$ as well as the arylamidase at 7.0 were high in cotyledons[7].

\section{Anti-Helmintic Activity}

It was suggested that crude alcoholic extract, ethyl acetate and methanol fractions of Clitoria ternatea substantially demonstrated paralysis and triggered fatality of worms particularly at concentration of $50 \mathrm{mg} /$ $\mathrm{ml}$, as contrasted to standard recommeded piperazine citrate. In another research study, flowers, leaves, stems and roots of Clitoria ternatea were examined for anti-helmintic activity on grown-up Indian earthworms Pheretima posthuma. Methanol extract of roots is most potent and needs very less time to paralyze and kill the worms, in comparison to other extracts. The potency of anthelmintic activity increases from flowers to leaves to stem to roots.

\section{Diuretic Activity}

When no significant effect was seen by implementation of oral administration, the powdered form of dried whole root and ethanol extract of Clitoria ternatea were assessed for diuretic activity by opting for Intravenous means. A single I.V. dose of the extract without affecting the volume of urine produced increased the urinary excretion of $\mathrm{Na}, \mathrm{K}$ and decrease in Cl.

\section{Anti-Microbial Activity}

The presence of small molecular weight, cystein rich protein, finotin acquired from seeds of the plant Clitoris ternatea has been identified for its antifungal property. The crude extract obtained from seeds of Clitoria ternatea exhibited a robust antifungal activity against the test fungi A. niger as well as A. ochraceous followed by various other microscopic organisms.

Anti-bacterial activity was carried out against Salmonella spp. and also Shigella dysenteriae, organisms responsible for triggering enteric fever. On top of that, the crude methanol extract revealed to have anti-bacterial activity against $\mathrm{K}$. pneumonia and $\mathrm{P}$. aeruginosa as well.

\section{Hepatoprotective Activity}

The paracetamol induced liver toxicity in mice treated with methanol extract of Clitoria ternatea obtained from the leaves, showed a remarkable decrease in ALT, AST, and bilirubin levels, all of which were 
elevated in group with paracetamol $(\mathrm{p}<0.01)$. The leaf extract of Clitoria ternatea is also found to be protective against histopathological alterations.

\section{Anti-Asthmatic Activity}

Clitoria ternatea root extract's anti asthmatic activity was assessed by preliminary phytochemical screening, intense toxicity studies and anti-asthmatic activity using milk induced leukocytosis and eosinophilia in mice, egg albumin induced mass cell degranulation in rats as well as passive cutaneous anaphylaxis in rats result revealed that the LD50 of ECTR is greater than $1300 \mathrm{mg} / \mathrm{kg}$.

\section{Cytotoxic Activity}

A research study of ethanolic extract of Clitoria ternatea verified that the plant holds cytotoxic as well as antioxidant activities. The extract revealed potent cytotoxic activity in trypan blue dye exclusion method utilizing DLA cell lines with EC50 values of $305 \mu \mathrm{g} / \mathrm{ml}$ and displayed a dose dependent decline in cell count for all the concentrations examined.

\section{Antioxidant Activity}

The antioxidant activity was examined by DPPH free radical method. The extract exhibited potent antioxidant activity with EC50 with value of $36.5 \mu \mathrm{g} / \mathrm{ml}$. There was a dose dependent rise in the antioxidant activity for all the examined concentrations.

As the antioxidant activity is commonly credited to phenolic substances within plants the total phenolic content within the aqueous extract and gel were determined. The IC50 values were $1 \mathrm{mg} / \mathrm{mL}$ and $4 \mathrm{mg} / \mathrm{mL}$, respectively.

\section{Immunomodulatory Activity}

SRBCs-sensitized rats were used to investigate the immunomodulatory activity of aqueous extract of seed and roots of Clitoria ternatea. The studies were conducted by the means of oral administration. The impacts on cell medicated resistance were examined by determining Delayed Type Hypersensitivity (DTH) action in SRBC-sensitized rats. The immunomodulatory activity of Clitoria ternatea can be attributed to decrease immune cell sensitization, immune cell presentation and phagocytosis. The anti-inflammatory and antioxidant properties maybe playing a crucial role in immunomodulatory activity.

\section{Wound Healing Activity}

Excision, incision and dead space models in rats were utilized in research studies of wound healing activity of seed and root extract of Clitoria ternatea. The oral administration and topical ointment method were employed, significant improvement in wound healing in excision, incision, and dead-space model was seen. The effects of seed and root extract were nearly comparable to that of cotrimoxazole ointment. The study also revealed that Clitoria ternatea affected all three phases of wound healing, inflammatory, proliferative and remodeling.

\section{Hypolipidemic Activity}

The anti-hyperlipidemic result of Clitoria ternatea L. was researched in experimentally induced hyperlipidemia in rats. In the investigation, the poloxamer 407 induced acute hyperlipidemia and diet-induced hyperlipidemia rat models were used. Significant reduction in levels of total serum cholesterol, triglycerides, very low-density lipoprotein cholesterol, were revealed by oral administration of hydroalcoholic extract of roots and seeds of Clitoria ternatea. The atherogenic index and the HDL:LDL ratio were also stabilized after treatment in diet-induced rats.

\section{Side Effects and Toxicity}

During research studies, the animals displayed decreased mobility, but no symptoms of convulsion or loss of writhing reflex were observed. This indicated that the Clitoria ternatea has low toxicity profile. Bacillus subtilis rec assay, Salmonella typhimurium Ames Test and micronucleus examination were employed to study the mutagenic effect of aqueous extract of Clitoria ternatea. The extract presented with negative outcomes in both bacterial as well as mammalian cell cultures, proving presence of no mutagenic activity. LD50 of ethanol extract of Clitoria ternatea root was more than $1300 \mathrm{mg} / \mathrm{kg}$ in mice. Acute oral toxicity study came up with the fact that there was no mortality up to $3000 \mathrm{mg} / \mathrm{kg}$ in mice. After a single dose of $1000 \mathrm{mg} / \mathrm{kg}$ in rats, no death or abnormal condition was seen within 72 hours. The same extract was found to be safe even at the dose of $2000 \mathrm{mg} / \mathrm{kg}$ body weight in rats. Surprisingly for ethanol extract of aerial parts of Clitoria ternatea there was no death observed in rats even at the dose of $2 \mathrm{~g} / \mathrm{kg}$.

\section{CONCLUSION}

Attention of the pharmaceutical sector is concentrated towards design, development and advancement of brand-new plant-based medicines with thorough examination of leads from Traditional system of medicines. In the research study of Clitoria ternatea alcoholic extract of roots, leaves as well as flowers offers various medicinal activities like anti-leprosy, anti-inflammatory, anti-helmintic, immunomodulatory, anti-asthmatic, anti-depressant, analgesic, antipyretic, antifungal, as well as antihyperlipidemic. Numerous vital phytoconstituents responsible for the activity were isolated. The clinical study on Clitoria ternatea suggests to have a huge biological potential in the plant. Though the reported evidences and studies sustains the safety and effectiveness of Clitoria ternatea, yet the quality of the evidences is not-enough with aspect to its bioactive secondary metabolites, bioavailability, pharmacokinetics and therapeutic value consisting of clinical trials, on which sufficient information is yet to be discovered. It is highly believed that comprehensive 
information as provided in this evaluation may offer comprehensive evidences for utilization of this plant in various medications and drug development. At the very same time, the natural available and aqueous extracts of Clitoria ternatea can be further exploited as a source of useful phytochemical compound for pharmaceutical market.

\section{REFERENCES}

1. Girish Kumar Gupta, Jagbir Chahal, Manisha Bhatia Maharishi Markandeshwar College of Pharmacy, Mullana, Ambala, Haryana-133203.

2. Prof Dr Ali Esmail Al-Snafi Department of Pharmacology, College of Medicine, Thi qar University, Nasiriyah, Iraq.

3. Chakraborty GS1, Kumar V1, Gupta S1, Kumar A1, Gautam N1, Kumari L11 Pharmacy Institute, Noida Institute of Engineering \& Technology, 19,
Knowledge Park-II, Greater Noida - 201306, Uttar Pradesh, India.

4. Al-Snafi AE. The chemical constituents and pharmacological effects of Calendula officinalis - A review. Indian Journal of Pharmaceutical Science \& Research. 2015; 5(3): 172185.

5. Morris JB. Legume genetic resources with novel value added industrial and pharmaceutical use. In: Janick J. (Ed.), Perspectives on new crops and new uses. ASHS Press, Alexandria, VA, USA. 1999: 196-201.

6. Kamilla L, Mansor SM, Ramanathan S and Sashidharan S. Antimicrobial activity of Clitoria ternatea (L.) extracts. Pharmacology online. 2009; 1: 731-738.

7. Anand SP, Doss A and Nandagopalan V. Antibacterial studies on leaves of Clitoria ternatea Linn.-A high potential medicinal plant. Int $\mathbf{J}$ Applied Bio Pharm Tech. 2011; 2(3): 453-456. 